\title{
Optimized Formation of Benzyl Isothiocyanate by Endogenous Enzyme and its Extraction from Carica Papaya Seed
}

\author{
Wen-Zhao Li, Yi-Deng Du, Yan Zhang, Ying-Zhou Chi, Zong-Yi Shi, Wen Chen, \\ Mei-Juan Ruan and Hua-Ping Zhu* \\ Key Laboratory of Food Nutrition and Safety (Tianjin University of Science and Technology), Ministry of Education, Tianjin \\ 300457, China \\ *For correspondence: Email: yideng126@126.com; Tel: +8615122396920
}

\begin{abstract}
Purpose: To use endogenous myrosinase in Carica papaya seed to convert benzyl glucosinolate (BG) to benzyl isothiocyanate (BITC) and then extract it for further studies.

Methods: Process variables including seed powder particle size, sample-to-solvent ratio, $\mathrm{pH}$ of buffer solution, enzymolysis temperature, enzymolysis time were investigated using single-factor experiments and response surface methodology coupled with Box-Behnken design for further optimization of conversion conditions. The formed benzyl isothiocyanate was extracted by steam distillation method and purified by thin-layer chromatography (TLC). Relevant process variables were also studied. Finally, the purified benzyl isothiocyanate was analyzed by gas chromatography-mass spectrometer (GC-MS) and compared to BITC standard.

Results: Optimum enzymolysis conditions were seed powder particle size, 90 - $120 \mu$ m; sample-tosolvent ratio, 1:20; pH of buffer solution, 4.8; enzymolysis temperature, 40 OC; and enzymolysis time, 27 min. Enzymolysis liquid was steam-distilled for $2 h$, extracted twice using dichloromethane (DCM) (using 3 times the volume of distillate each time) as extractant. Finally, 1:20 was adopted as the optimal dichloromethane-petroleum ether (DCM-PE) elution proportion to wash out the unnecessary compounds.

Conclusion: The result showed that benzyl isothiocyanate was formed under the optimized conditions and was no doubt the basic component of the extraction.
\end{abstract}

Keywords: Carica papaya, Benzyl isothiocyanate, Endogenous myrosinase, Enzymolysis, Extraction

Tropical Journal of Pharmaceutical Research is indexed by Science Citation Index (SciSearch), Scopus, International Pharmaceutical Abstract, Chemical Abstracts, Embase, Index Copernicus, EBSCO, African Index Medicus, JournalSeek, Journal Citation Reports/Science Edition, Directory of Open Access Journals (DOAJ), African Journal Online, Bioline International, Open-J-Gate and Pharmacy Abstracts

\section{INTRODUCTION}

Papaya seed has been used as vermifuge in India, Central and South America for centuries; however, most of the seeds are wasted during papaya processing [1]. David J Williams pointed that papaya is one of the few tropical fruit species to contain a glucosinolate [2]. Since most glucosinolate-containing plants possess 4-5 glucosinolates of which 1-2 predominate, the difference is in papaya seed benzyl glucosinolate (BG) appears to be the sole glucosinolate present [2].

It has been known for many years that papaya seed when macerated with water gives rise to the benzyl isothiocyanate (BITC) content, the enzymic hydrolysis product of BG [3]. 
Glucosinolates are metabolized to yield isothiocyanates by the assist of enzymes commonly called myrosinase (thio-glucoside hydrolases, EC 3.2.3.1) [3, 4], enzymes that are brought into contact with their substrate(s) upon damage to seeds [5]. Papaya seed extraction has been reported have anthelmintic properties [5], antimicrobial activity [6], antifungal activity [7], inhibition of cell proliferation [8], the potential to reduce the risk of various types of cancers which due, at least in part, to BITC.

Several methodologies have been reported concerning the extraction of BITC in Carica papaya seed [5], but to the best of our knowledge, no specific study has been published in which enzymolysis and conversion conditions were optimized. For this reason, the degree of conversion of BG to BITC and the extraction efficiency of BITC could vary depending on the conversion and extraction conditions. Since BITC is volatile, in this study, steam distillation was used for the first time in BITC extraction. In contrast to solvent extract and Soxhlet extraction method, steam distillation-extraction can get rid of the less volatile material and the purification process can get easier.

Therefore, the objective of this study was to develop an adequate enzymolysis system and extraction method to obtain BITC from papaya seed. A purification method was also established to obtain the pure BITC, which can used for further investigation in biological activity research and also determine the actual active compounds in papaya seed extract.

\section{EXPERIMENTAL}

\section{Materials and chemicals}

Carica papaya seeds were supplied by Hainan Standard Bio-Technique Co Ltd, Hainan Province, China. The fruit material was authenticated by Professor Wen-Zhao Li, College of Food Engineering and Biotechnology, Tianjin University of Science and Technology. A voucher specimen (TUST-UC317452) has been kept in the herbarium of Tianjin University of Science and Technology - for future reference. BITC standard (purity, $98 \%$; molecular weight, 149.2), was obtained from Sigma Aldrich Co LLC, St Louis, MO, USA; all other chemicals used were of analytical grade.

\section{Carica papaya seed samples preparation}

Carica papaya seed samples were collected from fresh Carica papaya in September and were dried under sunlight until water content up to 10 $\%$. Subsequently, they were packaged and stored at $4{ }^{\circ} \mathrm{C}$ until analysis.

\section{Conversion of benzyl glucosinolate (BG) to benzyl isothiocyanate (BITC)}

The convention of BG to BITC was done according to Rossetto [9]. Samples were prepared by grinding seeds with a grinder and 50 $\mathrm{g}$ powder was mixed with $50 \mathrm{ml}$ buffer solution $(\mathrm{pH} 7.0$ using $0.1 \mathrm{~mol} / \mathrm{l}$ citric acid and $0.2 \mathrm{~mol} / \mathrm{l}$ disodium hydrogen phosphate solution). The mixture was shaken in a Vortex device for $5 \mathrm{~min}$ and then was incubated at $35{ }^{\circ} \mathrm{C}$ in water bath for $2 \mathrm{~h}$.

To optimize the conversion of BG to BITC, single-factor experiments were conducted by varying most of the aforementioned parameters: (1) seed powder particle size $(90-120,120-150$, 150-180, 180-250, 250-380 $\mu \mathrm{m}$ ); (2) sample-tosolvent ratio(1:5, 1:10, 1:15, 1:20, 1:25); (3) $\mathrm{pH}$ of buffer solution (2, 3, 4, 5, 6, 7, 8); (4) enzymolysis temperature $\left(10,20,30,40,50^{\circ} \mathrm{C}\right)$; (5) enzymolysis time $(0,20,40,60,80 \mathrm{~min})$.

Response surface methodology (RSM) couple with Box-Behnken design (BBD) was used for further optimization of extraction condition [10]. A three-level three-factor factorial design was adopted to see the interaction effect of $\mathrm{pH}$ of buffer solution, enzymolysis temperature and enzymolysis time on the procedure of $B G$ to BITC in consideration of their significant influence on the activity of myrosinase.

\section{Preparation of seed extracts}

Seed extracts were prepared by steam distillation device. After BG was converted to BITC, the incubated mixture was transferred to steam distillation device. BITC was brought out by water steam as well as volatile oil and they were collected together. The distillation time was depends.

Dichloromethane (DCM) and several other kinds of solvents were used to extract BITC from distillate, the suitable volume and number of extract times was studied in this research. The final extract was concentrated by rotary evaporators in vacuum at $38{ }^{\circ} \mathrm{C}$.

\section{Purification of seed extracts}

The seed extracts, prepared as described above, and were loaded onto a silica gel (GF254, 10-40 $\mu \mathrm{m}$ mesh) plate $(10 \times 20 \mathrm{~cm}$, silica gel layer thickness $1.0 \mathrm{~mm}$ ). Dichloromethane (DCM) and 
petroleum ether (PE) were used as developing solvent and their proportion was studied in this research for better separation effect. Since BITC has ultraviolet absorption at $254 \mathrm{~nm}$, it can be observed on the silica gel plate under ultraviolet lamp in contrast to BITC standard developed under the same conditions. The silica gel that had BITC adsorbed on it was scraped and eluted with PE 3 times. The three PE fraction were pooled and was concentrated by rotary evaporators in vacuum at $38{ }^{\circ} \mathrm{C}$ until PE was removed. The residues was collected and stored at $-18{ }^{\circ} \mathrm{C}$ until its analysis.

Rate of flow $\left(R_{f}\right)$ was used to evaluate the separation effect of developing solvent. $R_{f}$ was calculated as in Eq 1.

$\mathrm{R}_{\mathrm{f}}=\mathrm{a} / \mathrm{b}$.

where $a$ is the migration distance of solute, $b$ is the migration distance of developing solvent as is shown in Fig. 1. According to experience, separation effect would be good when $R_{f}$ value is between 0.25 and 0.75 .

$R_{s}$ is another evaluation index used to evaluate the separation effect of developing solvent which is defined as in Eq 2.

$\mathrm{R}_{\mathrm{s}}=\mathrm{I}_{1} /\left[\mathrm{I}_{1}+\mathrm{I}_{2}\right]$

where $I_{1}, I_{2}$ is the distance marked in Fig. 1. According to experience, two solutes would be well separated when $R_{s}$ value is around 0.5 .

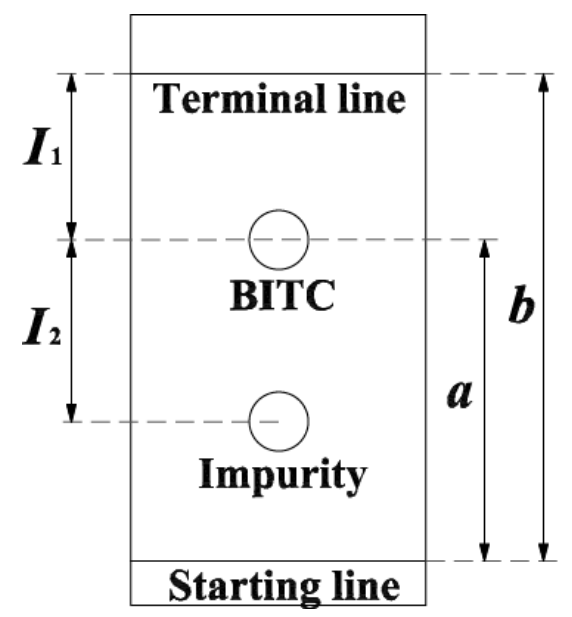

Fig 1: Illustration of thin-layer chromatography (TLC) plate

DCM and PE were used as developing solvent and their proportion was studied in this research for better separation effect.

\section{Measurement of BITC}

The measurement of BITC was done according to NY/T 1596-2008: thiourea colorimetry method
(Agricultural industry standard of the People's Republic of China). $50 \mu \mathrm{l}$ of sample was added to test tube with stopper mixed with $6 \mathrm{ml}$ of $80 \%$ ammonia alcohol. The test tube was shaken in a Vortex device for $5 \mathrm{~min}$ and then was incubated at $50{ }^{\circ} \mathrm{C}$ in water bath for $0.5 \mathrm{~h}$, and then cooling to room temperature. Then optical density values were measured at the wavelength of 235, 245, $255 \mathrm{~nm}$ in $10 \mathrm{~mm}$ quartz cuvette using ultravioletvisible spectrophotometer. Each test included blank controls containing $80 \%$ ammonia alcohol and triplicate test samples. Quantitative analysis was done by using the standard curve of BITC. The standard curve of BITC was as follows:

$\omega=\left\{O D_{245}-\left[\left(O_{235}+O D_{255}\right) / 2\right]\right\} \times 28.55$.

where $\omega$ is the concentration of BITC. $\mathrm{OD}_{235}$, $\mathrm{OD}_{245}, \mathrm{OD}_{255}$ are the optical density values at $235,245,255 \mathrm{~nm}$ respectively. For convenience we define OD as follow:

$\mathrm{OD}=\mathrm{OD}_{245}-\left[\left(\mathrm{OD}_{235}+\mathrm{OD}_{255}\right) / 2\right]$

Thus the concentration of BITC can be replaced by OD value for convenience in the following study.

\section{Confirmation of the BITC}

Aliquots of $1 \mu \mathrm{l}$ of BITC extract were injected in a Gas chromatography-Mass spectrometry (VARIAN model 4000) equipped with a DB-5 capillary column $(30 \mathrm{~m} \times 0.32 \mathrm{~mm}$ i.d, $0.25 \mu \mathrm{m}$ film thickness). The injection port was kept at 250 ${ }^{\circ} \mathrm{C}$, and a ramp temperature program was run at $50{ }^{\circ} \mathrm{C}$ (initial, and kept for $2 \mathrm{~min}$ ) increasing $10{ }^{\circ} \mathrm{C}$ $\mathrm{min}^{-1}$ to $250^{\circ} \mathrm{C}$ and kept for $5 \mathrm{~min}$. The split ratio was $10: 1$, and the helium carrier gas flow was $1.4 \mathrm{ml} \mathrm{min}{ }^{-1}$. The detection was done in a mass selective detector (VARIAN model 4000) with an ion source operating in $70 \mathrm{eV}$ and $200^{\circ} \mathrm{C}$. Fullscan GC-MS spectra were obtained by scanning from $\mathrm{m} / \mathrm{z} 40$ to 600 . BITC standard and the extracts of papaya seed whose myrosinase was inactivated were also detected under the same GC-MS conditions.

\section{Statistical analysis}

All analyses were carried out in triplicate, and the results expressed as mean \pm standard deviation. Significant difference was tested using multiple comparisons with the aid of MATLAB (version 7.12, MathWorks, Inc.). Response surface methodology coupled with Box-Behnken design was done using Design-Expert (version8.0.6, Stat-Ease, Inc.). Figures were draw using OriginPro (version 9, OriginLab Corporation). 


\section{RESULTS}

\section{The conversion of BG to BITC}

The results of single-factor experiments were summarized in Fig 2 (a-e). The difference significance test was done on 0.05 levels.

With regard to selecting the seed powder particle size (Fig 2a); the highest BITC content was detected at 90-120 $\mu \mathrm{m}$. It was also concluded that 1:20 was the most suitable sample-tosolvent ratio for the conversion of $B G$ to BITC. Subsequently, $\mathrm{pH} 4.0,30{ }^{\circ} \mathrm{C}$ and $20 \mathrm{~min}$ were selected as the optimal value.

The number of experiments required was 17 using a Box-Behnken experimental for a threelevel three-factor factorial design, as shown in Table 1 together with the obtained results.
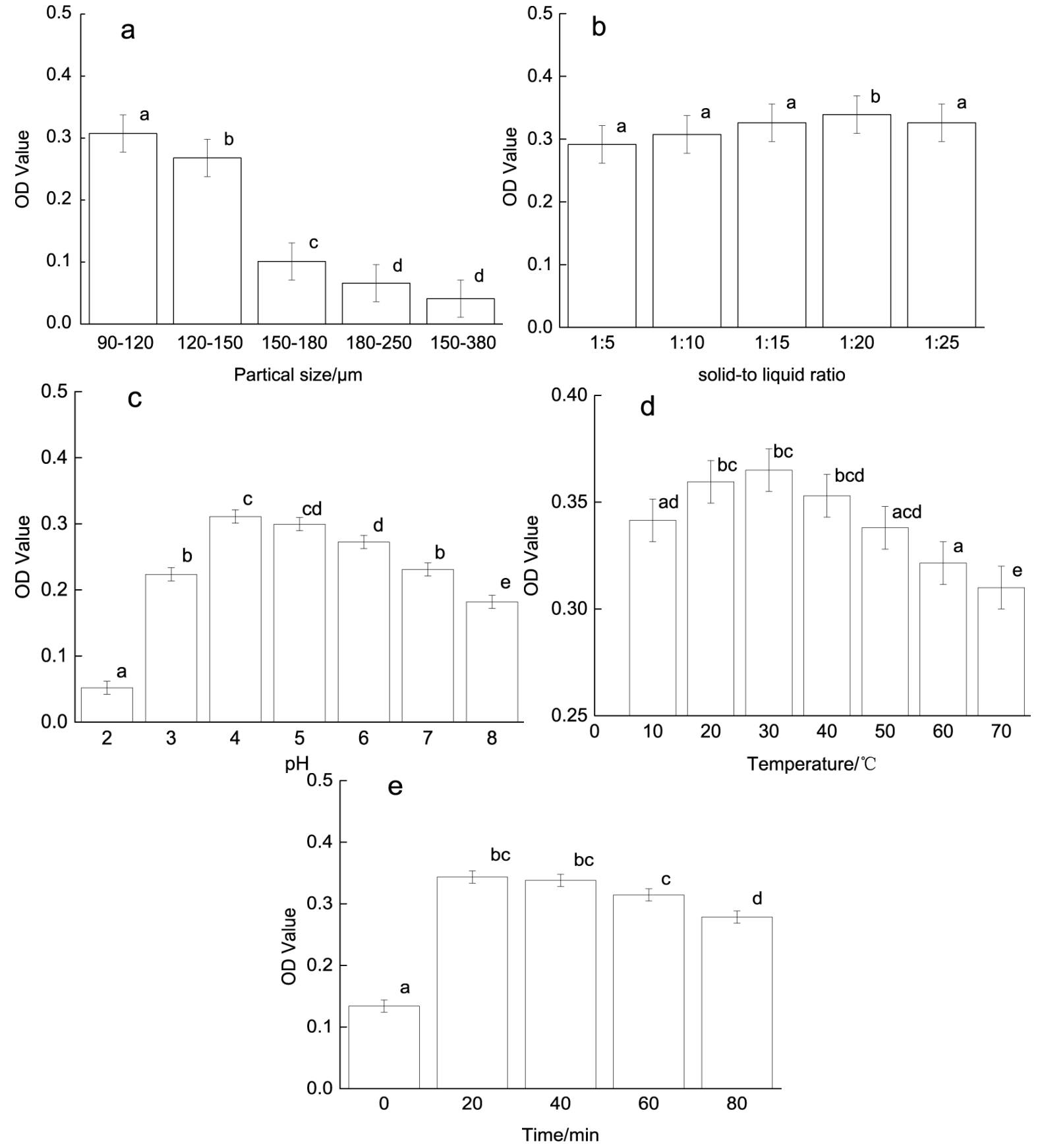

Fig 2: Effect of various parameters on the conversion of BG to BITC: (a) seed powder particle size; (b) sampleto-solvent ratio; (c) pH of buffer solution; (d) enzymolysis temperature; (e) enzymolysis time 
Table 1: Box-Behnken design scheme and corresponding results

\begin{tabular}{llccc}
\hline No. & $\mathbf{p H}$ & Time $(\mathbf{m i n})$ & Temperature $\left({ }^{\circ} \mathbf{C}\right)$ & Absorbance \\
\hline 1 & 5.5 & 35 & 40 & 0.35 \\
2 & 4.0 & 35 & 30 & 0.37 \\
3 & 2.5 & 35 & 20 & 0.21 \\
4 & 4.0 & 55 & 40 & 0.32 \\
5 & 4.0 & 15 & 40 & 0.34 \\
6 & 4.0 & 15 & 20 & 0.33 \\
7 & 5.5 & 55 & 30 & 0.28 \\
8 & 2.5 & 15 & 30 & 0.17 \\
9 & 5.5 & 15 & 30 & 0.34 \\
10 & 2.5 & 35 & 40 & 0.17 \\
11 & 4.0 & 35 & 30 & 0.36 \\
12 & 2.5 & 55 & 30 & 0.17 \\
13 & 4.0 & 55 & 20 & 0.34 \\
14 & 4.0 & 35 & 30 & 0.37 \\
15 & 4.0 & 35 & 30 & 0.35 \\
16 & 4.0 & 35 & 30 & 0.36 \\
17 & 5.5 & 35 & 20 & 0.33 \\
\hline
\end{tabular}

Table 2: Analysis of variance for the second-order regression model

\begin{tabular}{lcccccc}
\hline Factor & SS & df & MS & $\boldsymbol{F}$ & $\boldsymbol{P}$ & Signifiance \\
\hline Model & 0.089 & 9 & $9.89 \mathrm{E}-003$ & 112.09 & $<0.0001$ & $* * *$ \\
A-pH & 0.043 & 1 & 0.043 & 484.09 & $<0.0001$ & $* * *$ \\
B-time & $6.23 \mathrm{E}-004$ & 1 & $6.23 \mathrm{E}-004$ & 7.06 & 0.0326 & $*$ \\
C-temperature & $6.38 \mathrm{E}-005$ & 1 & $6.38 \mathrm{E}-005$ & 0.72 & 0.4233 & $*$ \\
AB & $8.79 \mathrm{E}-004$ & 1 & $8.79 \mathrm{E}-004$ & 9.96 & 0.0160 & $*$ \\
AC & $1.15 \mathrm{E}-003$ & 1 & $1.14 \mathrm{E}-003$ & 13.01 & 0.0087 & $* *$ \\
BC & $1.99 \mathrm{E}-004$ & 1 & $1.99 \mathrm{E}-004$ & 2.26 & 0.1765 & $*$ \\
$\mathrm{~A}^{2}$ & 0.038 & 1 & 0.038 & 431.55 & $<0.0001$ & $*$ \\
$\mathrm{~B}^{2}$ & $3.47 \mathrm{E}-003$ & 1 & $3.47 \mathrm{E}-003$ & 39.40 & 0.0004 & $*$ \\
$\mathrm{C}^{2}$ & $6.82 \mathrm{E}-005$ & 1 & $6.82 \mathrm{E}-005$ & 0.77 & 0.4085 & \\
Residual & $6.18 \mathrm{E}-004$ & 7 & $8.82 \mathrm{E}-005$ & & & \\
Lack of fit & $3.85 \mathrm{E}-004$ & 3 & $1.28 \mathrm{E}-004$ & 2.20 & 0.2302 & \\
Pure error & $2.33 \mathrm{E}-004$ & 4 & $5.82 \mathrm{E}-005$ & & & \\
Total SS & 0.090 & 16 & $9.89 \mathrm{E}-003$ & & & \\
\hline
\end{tabular}

Note: * means $p<0.05 ;{ }^{* *}$ means $p<0.01 ;{ }^{* * *}$ means $p<0.0001$

The experimental data were fitted into the second-order polynomial equations and the regression coefficients were calculated. The relation among the variables (as coded values) and the analytical signal (OD value) was fitted using equation 5 :

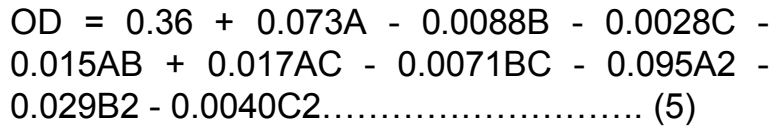

The significance of the coefficients of the model was determined by analysis of variance as summarized in Table 2.

Eq 3 together with the ANOVA is used to find the direction in which the variables should be changed in order to optimize the conversion efficiency. It should be mentioned that in Eq 3 the non-significant variables $(p>0.05)$ were discarded in order to improve the model.
Three-dimensional (3D) response surface plots are reported as a function of two factors, maintaining all other factors at fixed levels, and are more helpful in understanding both the main and the interaction effects of these two factors. $3 \mathrm{D}$ response surface plots (Fig $3 a-f)$ were based on the model equation (Eq 3).

The effect of $\mathrm{pH}$ of buffer solution and enzymolysis time on absorbance value is shown in Fig. 3(a, b). The data shows that the maximum absorbance is obtained at medium values of time and greater values of $\mathrm{pH}$. It can be seen from the Fig. 3(a, b) that the influence of $\mathrm{pH}$ on the absorbance value is more significant than enzymolysis time which is consistent with the analysis of variance for the second-order regression model. Moreover the absorbance values increase significant during $\mathrm{pH} 2.5$ to 4.0 while stable during $\mathrm{pH} 4.0$ to 5.5 . The relation between the effects of the other variables on absorbance is plotted in Fig. 3(c-f). 
a

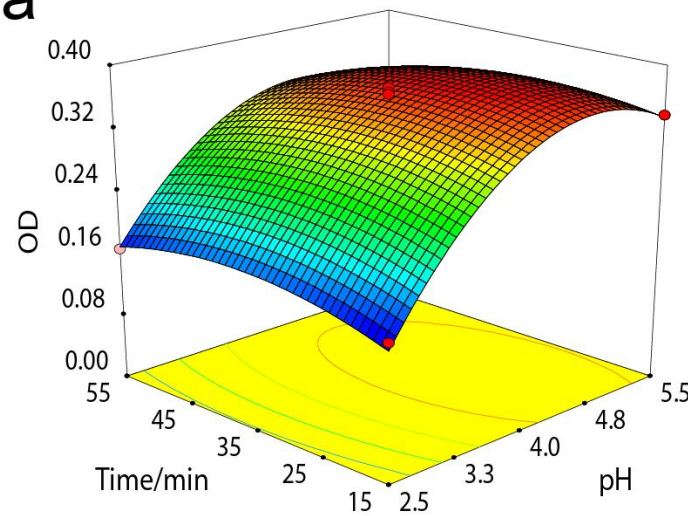

C
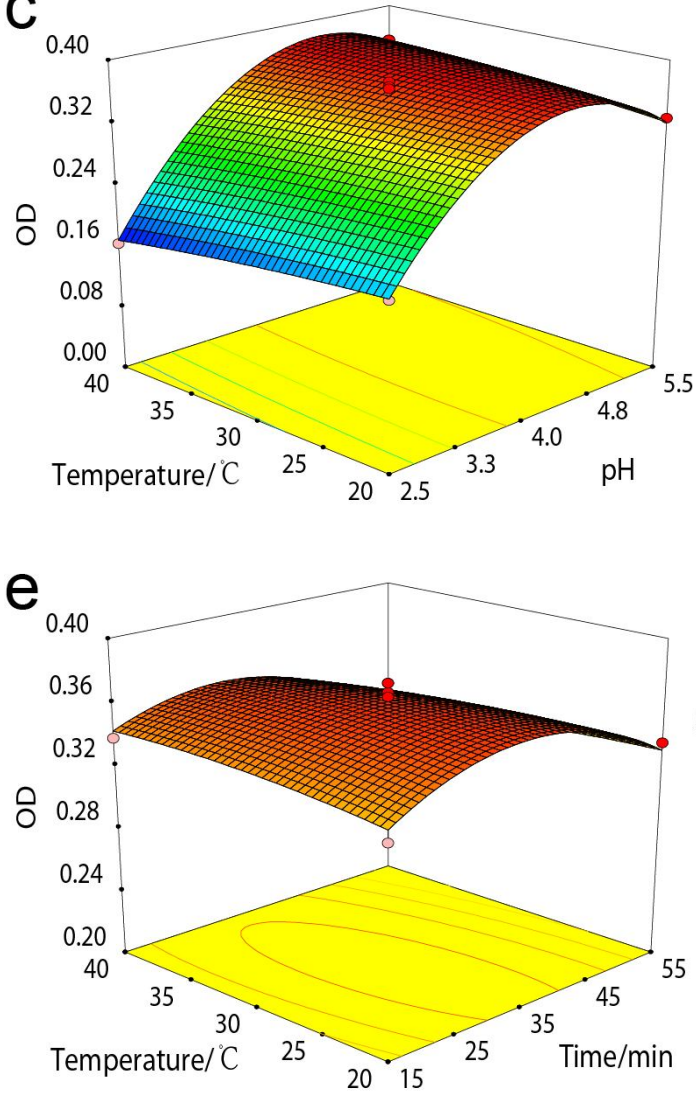

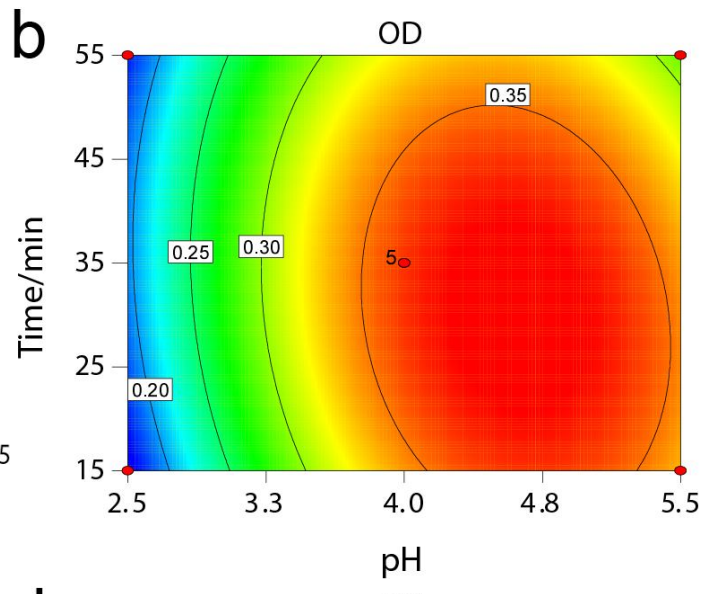

$\mathrm{OD}$

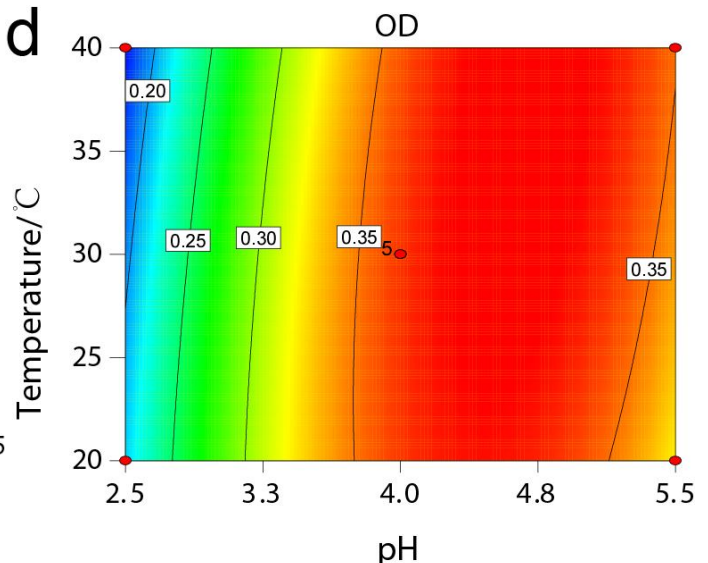

$f$

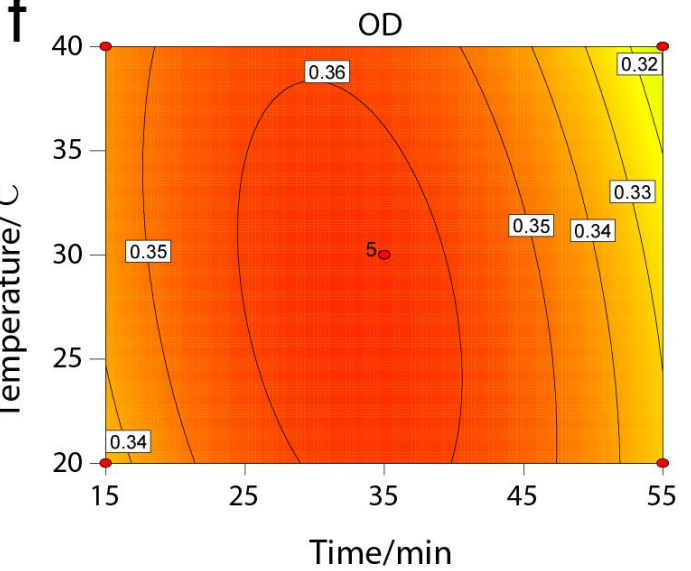

Fig 3: Response surface and contour plots for the effects of variables on the absorbance values of the seed extraction: enzymolysis time and $\mathrm{pH}$ of buffer solution $(\mathrm{a}, \mathrm{b})$; enzymolysis temperature and $\mathrm{pH}$ of buffer solution (c, d); enzymolysis temperature and enzymolysis time $(e, f)$

\section{Preparation of seed extracts}

Seed extracts were prepared by steam distillation device. With regard to selecting the proper distillation time, the highest OD value was detected at the third hour (result not shown). For environment-friendly thinking $2 \mathrm{~h}$ distillation time is enough to get most of the BITC out the enzymolysis liquid.
DCM and several other kinds of solvents were used to extract BITC from distillate. The result showed that PE, Hex, EtAc had comparable extract ability while DCM had significant higher extract rate (result not shown). It was decided that the experiments would be continued with DCM as extractant.

Subsequently, the influence of the volume and number of extract times were tested (Fig 4). It was found that 2 times was enough to obtain the 
sufficient BITC out of the distillate, since there was nearly no BITC extracted during the third time extraction. With regard to extractant volume, 1:3 (distillate: extractant solvent) showed better result as can be seen in Fig 4 .

In summary, the optimized extraction process was as follow. Enzymolysis liquid was distilled for two hours through water steam, then extract two times use DCM (three times the volume of distillate each time) as extractant. The final extract in DCM was gathered and concentrated by rotary evaporators.

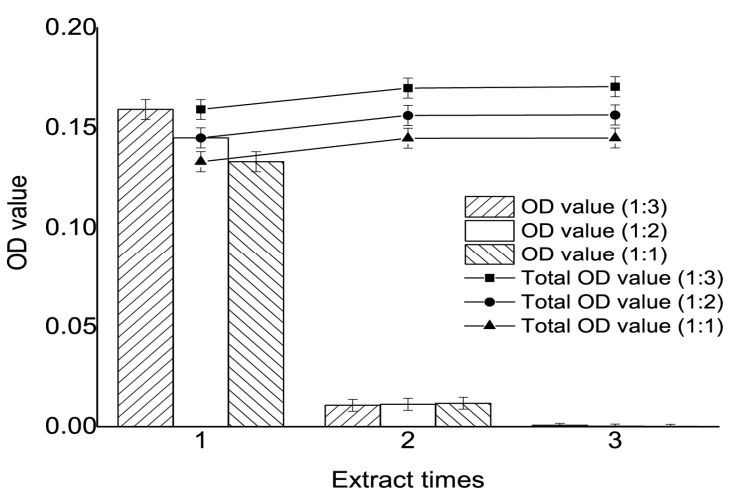

Fig 4: Effect of solvent usage and number extraction times on absorbance values of seed extraction

\section{Purification of seed extracts}

With regard to select the most suitable developing solvent, several different proportion of
DCM-PE solvent systems were studied, the result was shown in table 3 . In consideration of $R_{f}$ value (0.25 - 0.75), 1:20 and 1:30 of DCM: PE proportion is suitable; taking $R_{S}$ value into account, the $R_{s}$ value of 1:20 of DCM: PE proportion is closer to 0.5 . Finally $1: 20$ was adopted as the optimal DCM-PE elution proportion to wash out the unnecessary compounds.

Table 3: Effect of different proportions in the DCM-PE solvent system on BITC purification

\begin{tabular}{lll}
\hline $\mathbf{D C M}: \mathbf{P E}$ & $\mathbf{R}_{\mathbf{f}}$ & $\mathbf{R}_{\mathbf{s}}$ \\
\hline $1: 0$ & 1.00 & 1 \\
$1: 5$ & 0.85 & 0.38 \\
$1: 10$ & 0.78 & 0.42 \\
$1: 20$ & 0.67 & 0.52 \\
$1: 30$ & 0.55 & 0.63 \\
$0: 1$ & 1 & 1 \\
\hline
\end{tabular}

\section{Confirmation of BITC}

The confirmation of the BITC was done by comparing to BITC standard using GC-MS analysis. The most significant peak in the total ion chromatogram was identified at $14.1 \mathrm{~min}$ which was consistent with the retention time of BITC standard. The mass spectrum at $14.1 \mathrm{~min}$ was identical to the mass spectrum of BITC.

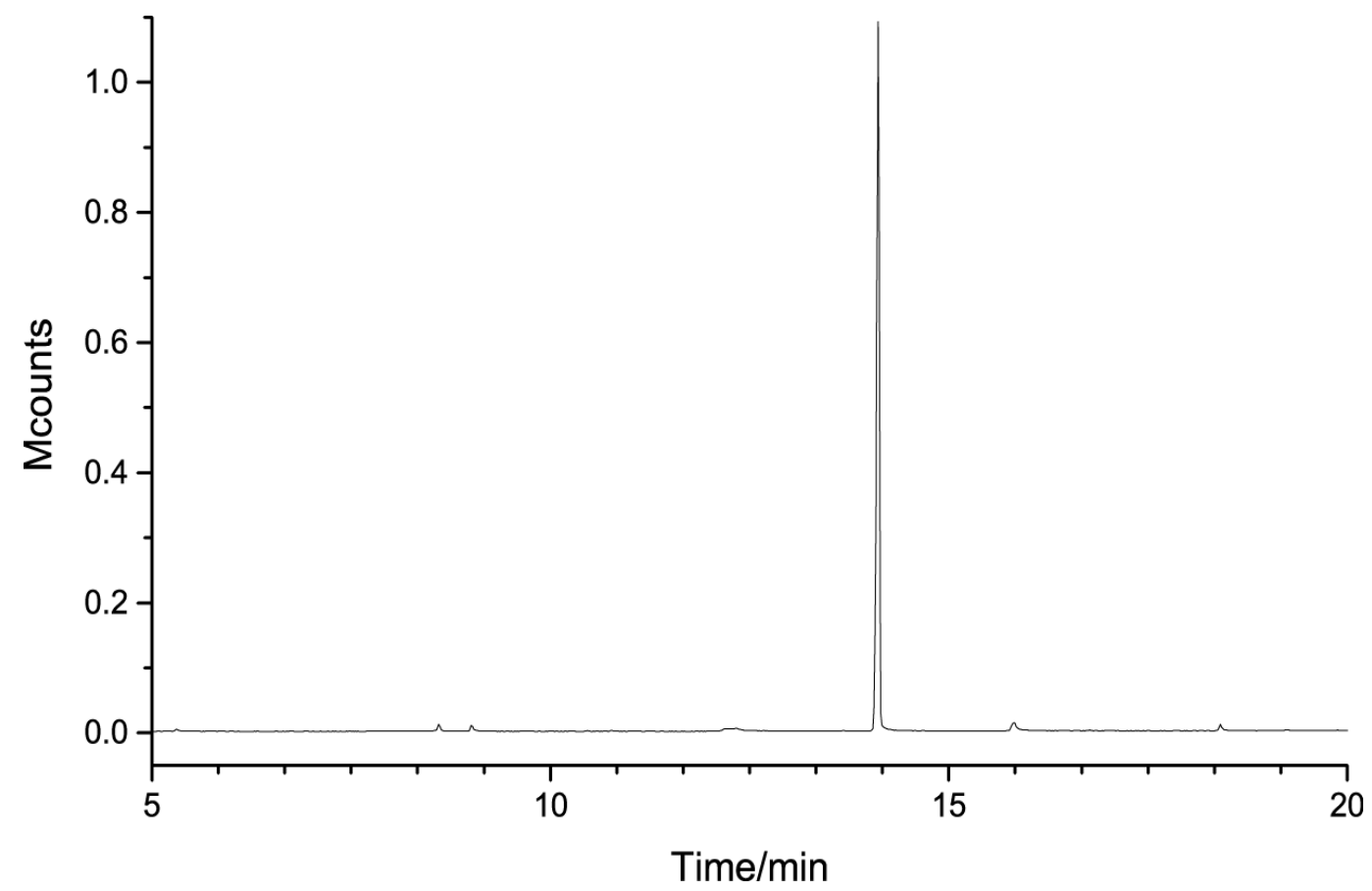

Fig 5: Total ion chromatogram of purified BITC sample extracted from papaya seed 


\section{DISCUSSION}

Although the extraction of BITC in Carica papaya seed have been reported, no specific study concerning enzymolysis and conversion conditions were published. The result showed that seed powder particle size, $\mathrm{pH}$ of buffer solution, enzymolysis time all has significant influence on the degree of conversion of BG to BITC. If papaya seed was not fully crushed, myrosinase and benzyl glucosinolate would not be in full contact, and thus less BG would be converted to BITC.

It has been reported that the $\mathrm{pH}$ of buffer solution has significant influence on the enzymatic degradation of glucosinolates and isothiocyanates tend to be generated in the neutral $\mathrm{pH}$ condition. In our experiment, $\mathrm{pH} 4.0$ was found to the benefit of BITC yield, take interaction effects into account, it is $\mathrm{pH}$ 4.8. The difference can be explained by the stability of different isothiocyanates. BITC is stable in acid environment while other isothiocyanates would be converted to thiocynate, epithionitriles, nitrile, oxazolidine-2-thinoe and so on. Thus the enzymatic degradation of glucosinolates was the combined action of myrosinase and $\mathrm{pH}$ environment. Since BITC is volatile, long heating time is not benefit for the reserve of it. As seen in Fig 2e, after 20 minutes the BITC yield was declined because the volatilization rate was faster than the generation rate.

In summary, after the evaluation of the main factors and their interactions, the following amounts were chosen as optimum conditions: seed powder particle size, $90-120 \mu \mathrm{m}$, sampleto-solvent ratio 1:20; $\mathrm{pH}$ of buffer solution, 4.8; enzymolysis temperature, $40{ }^{\circ} \mathrm{C}$; and enzymolysis time, 27 min. Under optimum conditions, the yield of BITC was $1.35 \%$. Finally, the optimized enzymolysis conditions have been demonstrated to be useful for conversion of $B G$ to BITC with the highest purity of BITC. This finding could be of interest to researchers as it is the first time that conversion of BG to BITC based on Carica papaya seed has been undertaken.

BITC is a non-polar substance and in the previously mentioned reports it was all extracted by organic solvent. Steam distillation was used for the first time to extract BITC for its volatile property, thus BITC and volatile oil would be extracted while non-volatile oil and other impurities would be excluded.
In contrast to BITC standard, it can be confirmed that the purified extract was BITC. It must be pointed out that barely no BITC detected in the extract of papaya seed whose myrosinase was inactivated (result no shown) which means that the BITC in the purified extract was generated under optimum conditions.

\section{CONCLUSION}

In this study, endogenous myrosinase in Carica papaya seed has been successfully used to convert benzyl glucosinolate to benzyl isothiocyanate and the process variables determined. Response surface methodology coupled with Box-Behnken design has been successfully applied to optimize the conversion conditions.

\section{ACKNOWLEDGEMENT}

This research was supported by the National Science-Technology Pillar Program (grant no.2012BAD33B09 and 2012BAD31B06). We gratefully acknowledge Modern Analytical Technology Research Center of Tianjin University of Science and Technology for GCMS analysis. We also thank Hainan Standard Bio-Technique Co. Ltd for supplying the papaya seed used.

\section{REFERENCES}

1. Fahey JW, Zalcmann AT, Talalay $P$. The chemical diversity and distribution of glucosinolates and isothiocyanates among plants. Phytochemistry, 2001; 56(1): 5-51.

2. Williams DJ, Pun S, Chaliha $M$, Scheelings $P$, O'Hare $T$. An unusual combination in papaya (Carica papaya): The good (glucosinolates) and the bad (cyanogenic glycosides). J Food Compos Anal, 2013; 29(1): 8286.

3. Chávez-Quintal Pedro, González-Flores Tania, Rodríguez-Buenfil Ingrid, Gallegos-Tintoré Santiago. Antifungal Activity in Ethanolic Extracts of Carica papaya L. cv. Maradol Leaves and Seeds. Indian J Microbial, 2011; 51(1): 54-60.

4. Prashar A, Siddiqui F, Singh AK. Synthetic and green vegetable isothiocyanates target red blood leukemia cancers. Fitoterapia, 2012; 83(2): 255-265.

5. Ettlinger MG, Hodgkins JE. The mustard oil of papaya seed. J Org Chem, 1956; (21): 204-205.

6. Rossetto MRM, do Nascimento JRO, Purgatto E, Fabi $J P$, Lajolo FM, Cordenunsi BR. Benzylglucosinolate, Benzylisothiocyanate, and Myrosinase Activity in Papaya Fruit during Development and Ripening. J Agric Food Chem, 2008; 56(20): 9592-9599. 
7. Kermanshai R, McCarry BE, Rosenfeld J, Summers PS, Weretilnyk EA, Sorger GJ. Benzyl isothiocyanate is the chief or sole anthelmintic in papaya seed extracts. Phytochemistry, 2001; 57(3): 427-435.

8. Radulovic NS, Dekic MS, Stojanovic-Radic ZZ. Antimicrobial volatile glucosinolate autolysis products from Hornungia petraea (L.) Rchb. (Brassicaceae). Phytochem Lett, 2012; 5(2): 351-357.
9. Palmieri S, Leoni O, lori R. A steady state kinetics study of myrosinase with direct UV spectrophotometric assay. Anal Biochem, 1982; (123): 320-324.

10. Ares AM, Bernal J, Martin MT, Bernal JL, Nozal MJ. Optimized Formation, Extraction, and Determination of Sulforaphane in Broccoli by Liquid Chromatography with Diode Array Detection. Food Anal Methods, 2014; 7(3): 730-740. 